\section{The great (mouse) depression}

\section{By Tim Fulmer, Staff Writer}

Preclinical models of depression are generally unable to account for genetic variations that predispose certain people to the disorder. Consequently, proof of efficacy in animal models of depression is even less predictive of efficacy in humans than it is for many other indications. A group at Duke University now is offering a strategy for designing animals with genetic vulnerability to depression. ${ }^{1}$

As reported last month in The Proceedings of the National Academy of Sciences, Jean-Martin Beaulieu and colleagues engineered mice to express the murine equivalent of a rare SNP in a gene that codes for tryptophan hydroxylase 2 (TPH2), one of two enzymes in the serotonin (5-HT) biosynthetic pathway. Beaulieu was formerly at Duke and is now assistant professor and Canadian research chair in molecular psychiatry at the Centre de recherche de l'Université Laval RobertGiffard (CRULRG).

A previous study by the researchers showed that in a cell culture assay, the SNP resulted in an $80 \%$ loss of enzyme function. Moreover, the SNP had a significantly higher frequency in a cohort of 87 patients with unipolar major depression compared with controls without a history of mental illness $(p<0.001){ }^{2}$

The Duke group engineered the SNP into the equivalent site of the murine $\mathrm{TPH} 2$ gene and studied its impact on serotonin signaling pathways and behavior. The resulting heterozygous and homozygous mutant mice had significant reductions in 5-HT synthesis rates and 5-HT tissue content in the striatum, frontal cortex and hippocampus compared with wild-type controls $(p<0.012)$.

In addition, glycogen synthase kinase-3 $\beta$ (GSK-3 $\beta$ ) activity was significantly increased in the frontal cortex of the homozygous mice compared with wild-type mice $(p<0.006)$. GSK-3 $\beta$ is involved in neuronal cell development and has a role in mood disorders and schizophrenia. The mood stabilizer lithium is a direct inhibitor of the enzyme. ${ }^{3}$

In the PNAS paper, the knock-in mice had altered 5-HT signaling in the brain that correlated with aberrant neurobehavioral responses. In two standard tests for neurobehavioral abnormalities, the tail-suspension test and the dark-light emergence test, the TPH2 mutant mice showed behavioral aberrations that were reversible through treatment with thiadiazolidinone TDZD-8, a generic GSK-3 $\beta$ inhibitor.

The authors concluded that the mice "represent a unique animal model to study the biological functions of TPH2 and brain 5-HT."

Beaulieu told SciBX that the PNAS paper suggests a "global strategy" for generating knock-in mice that could potentially address human genetic susceptibility to psychiatric disorders. The strategy involves identifying a SNP in human populations that confers disease risk, engineering the equivalent SNP into mice, measuring the biochemical and behavioral consequences and ultimately finding therapeutic targets.

He noted that such an approach generally does require a high degree of structural and functional homology between the proteins that comprise murine and human neuronal signaling pathways. In the case of TPH2, the interspecies amino acid identity is $92 \%$.

John Cryan, senior lecturer in pharmacology and principal investigator at University College Cork, told SciBX that the new model could potentially affect the depression space "in much the same way as rare familial mutations have led to the development of very important transgenic models of Alzheimer's disease, which have revolutionized drug discovery in that area."

Going forward, Beaulieu's group is using the TPH2 knock-in mice to investigate the effects of chronic antidepressant treatment with approved selective serotonin reuptake inhibitors such as Prozac fluoxetine from Eli Lilly and Co. and Paxil paroxetine from GlaxoSmithKline plc.

Another direction for potential future research that interests Beaulieu is knocking multiple SNPs into mice to get an even better proxy for human depression. That could be an important step, said Mark Epping-Jordan,

CSO and cofounder of Addex Pharmaceuticals S.A., because, "in general, it is not possible to comprehensively model complex human diseases like depression or schizophrenia in rodents-instead, specific symptoms of disease are modeled."

Addex has two series of negative allosteric modulators that target undisclosed molecules and are in preclinical development to treat depression. The company also is developing metabotropic glutamate receptor 2 (mGluR2) positive allosteric modulators for schizophrenia and anxiety with Ortho-McNeil Pharmaceutical Inc., a Johnson \& Johnson company.

Gerard Marek, CSO for psychiatric biology at Lilly, said that "it would be particularly interesting to probe this model with relatively novel treatments such as lamotrigine or olanzapine/fluoxetine for bipolar depression. In addition to basic validation from a pharmacological perspective, such work might have implications for better relating the present transgenic model to distinct clinical subpopulations."

Lilly markets Symbyax, a combination of fluoxetine and the monoaminergic receptor antagonist olanzapine, to treat depressive episodes 
associated with bipolar disorder. GlaxoSmithKline markets Lamictal lamotrigine for bipolar I disorder and as an antiepileptic.

According to Epping-Jordan, "the explicit link between a known human mutation, deficient 5-HT biosynthesis and altered GSK-3 $\beta$ signaling, and the resulting phenotype, are novel features of the model."

In general, however, he noted that constitutive knock-in models have a basic limitation. "There may be adaptations that occur during development which may also contribute to the observed phenotype and may influence the effects of drugs in the model," said Epping-Jordan.

In response, Beaulieu noted that "the phenotype of humans with functional TPH2 partial loss-of-function mutations will also be a composite of the effect of reduced $\mathrm{TPH} 2$ activity both in adults and during development. So, in this case, our model may be more representative of a clinically relevant situation than a more typical inducible knockout model would be, in that ours should reproduce both the developmental and the nondevelopmental effects of the mutation."

That being said, he added, "this criticism may apply to the rescue of the TPH 2 mice phenotype using GSK-3 $\beta$ haploinsufficient mice.
This is why we confirmed our behavioral observations in these mice by using a GSK-3 $\beta$ inhibitor as well."

\section{REFERENCES}

1. Beaulieu, J., et al. Proc. Natl. Acad. Sci. USA; published online Jan. 22, 2008; doi:10.1073/pnas.0711496105

Contact: Marc G. Caron, Mouse Behavioral and Neuroendocrine Analyses Core Facility, Duke University Medical Center, Durham, N.C. e-mail: m.caron@cellbio.duke.edu

2. Zhang, X. et al. Neuron 45, 11-16 (2005)

3. Jope, R. \& Roh, M. Curr. Drug Targets 7, 1421-1434 (2006)

COMPANIES AND RESEARCH INSTITUTIONS MENTIONED

Addex Pharmaceuticals S.A. (SWX:ADXN), Geneva, Switzerland Centre de recherche de l'Université Laval Robert-Giffard (CRULRG), Beauport, Quebec, Canada

Duke University, Durham, N.C.

Eli Lilly and Co. (NYSE:LLY), Indianapolis, Ind.

GlaxoSmithKline plc (LSE:GSK; NYSE:GSK), London, U.K.

Johnson \& Johnson (NYSE:JNJ), New Brunswick, N.J.

Ortho-McNeil Pharmaceutical Inc., Raritan, N.J.

University College Cork, Cork, Ireland 